\title{
Converse piezoelectricity and ferroelectricity in crystals of lysozyme protein revealed by piezoresponse force microscopy
}

\author{
A. Stapleton, M. S. Ivanov, M. R. Noor, C. Silien, A. A. Gandhi, T. Soulimane, A. \\ L. Kholkin \& S. A. M. Tofail
}

To cite this article: A. Stapleton, M. S. Ivanov, M. R. Noor, C. Silien, A. A. Gandhi, T. Soulimane, A. L. Kholkin \& S. A. M. Tofail (2018) Converse piezoelectricity and ferroelectricity in crystals of lysozyme protein revealed by piezoresponse force microscopy, Ferroelectrics, 525:1, 135-145, DOI: 10.1080/00150193.2018.1432825

To link to this article: https://doi.org/10.1080/00150193.2018.1432825

曲 Published online: 15 Mar 2018.

Submit your article to this journal $₫$

Џ Article views: 55

View Crossmark data ¿ 


\title{
Converse piezoelectricity and ferroelectricity in crystals of lysozyme protein revealed by piezoresponse force microscopy
}

\author{
A. Stapleton ${ }^{a, b}$, M. S. Ivanov ${ }^{c}$, M. R. Noor ${ }^{b, d}$, C. Silien ${ }^{a, b}$, A. A. Gandhi ${ }^{a, b}$, T. Soulimane ${ }^{b, d}$, \\ A. L. Kholkin ${ }^{\mathrm{e}, \mathrm{f}}$, and S. A. M. Tofail ${ }^{\mathrm{a}, \mathrm{b}}$ \\ ${ }^{\mathrm{a} D}$ Department of Physics and Energy, University of Limerick, Limerick, Ireland; ${ }^{\mathrm{b}}$ Bernal Institute, University of \\ Limerick, Limerick, Ireland; 'CFisUC, Department of Physics, University of Coimbra, Coimbra, Portugal; ${ }^{\mathrm{d}}$ Chemical \\ and Environmental Science Department, University of Limerick, Limerick, Ireland; ${ }^{\mathrm{e}} \mathrm{CICECO}$ - Materials Institute

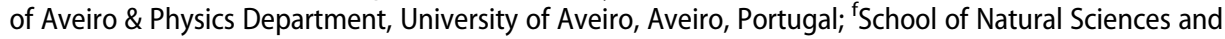 \\ Mathematics, Ural Federal University, Ekaterinburg, Russia
}

\begin{abstract}
This work investigates the converse piezoelectric effect in crystals of the protein lysozyme using Piezoresponse Force Microscopy (PFM) in contact and Hybrid modes. The mechanical properties of lysozyme crystals were mapped at the surface by means of Hybrid mode. In addition, ferroelectric loops were measured by the switchingspectroscopy PFM method (SS-PFM). We explore these findings using crystallographic principles and propose that the presence of defects within the crystal may lower the symmetry of lysozyme to a polar one. Our findings point towards the potential of exploiting lysozyme and other proteins in technical applications, especially those in which biocompatibility is critical.
\end{abstract}

\section{ARTICLE HISTORY}

Received 30 August 2017

Accepted 27 November 2017

\section{Introduction}

In recent years, PFM has been used to investigate the piezoelectric and ferroelectric behavior of many biological materials including amino acids [1, 2], engineered peptide nanotubes $[3,4]$, and viruses [5]. In the classical sense, piezoelectricity can only occur in materials with a non-centrosymmetric structure. The direct piezoelectric effect - the ability of some materials to generate electricity under stress - was discovered by the Curie brothers in 1880 [6]. A converse piezoelectric effect also exists; it is the ability of a material to deform under an electrical potential. A subset of piezoelectric materials are pyroelectric, possessing a spontaneous polarization that allows them to generate electricity when their temperature changes. Of the 32 crystallographic point groups, 21 lack a center of symmetry and 20 are piezoelectric [7]. Ten of the piezoelectric point groups are classed as polar (pyroelectric) and eleven as chiral. A reversible polarization exists in materials, where the spontaneous polarization assumes two opposite but thermodynamically equivalent states. This polarization can be switched under an externally applied field, which means that the material is ferroelectric.

CONTACT A. L. Kholkin kholkin@ua.pt

Color versions of one or more of the figures in the article can be found online at www.tandfonline.com/gfer. 
In terms of protein piezoelectricity, studies have been confined to the fibrous protein type. For example, piezoelectricity has been studied extensively in collagen [8-11]. To a lesser extent, piezoelectricity and ferroelectricity have been investigated in elastin $[12,13]$. A comprehensive understanding of piezoelectricity and ferroelectricity in non-fibrous proteins is lacking. Recently, we have reported evidence of the converse piezoelectric effect using PFM in monoclinic crystals of lysozyme [14].

Lysozyme is a globular protein found abundantly in hen egg whites as well as in mammalian secretions. It is one of the most well studied proteins and notably it can be crystallized in several different forms [15]. Like all natural protein crystals, crystals of lysozyme are noncentrosymmetric, thus satisfying the fundamental prerequisite of piezoelectricity. Tetragonal crystals of lysozyme are generally described by point group 422 . The piezoelectric tensor for crystals in this point group is limited to the shear piezoelectric coefficients $d_{14}$ and $-d_{14}$ [7].

Previously, Danielewicz-Ferchmin et al. have proposed that lysozyme in solution may demonstrate electrostriction and piezoelectric effects [16] based on reports that the hydration water density of lysozyme changes at different applied pressures [17]. Kalinin et al. have used PFM to investigate piezoelectricity in amyloid lysozyme fibrils adsorbed onto mica [18]. Here, we measure and quantify the converse effect in individual tetragonal crystals of lysozyme using PFM. Both contact and hybrid modes of PFM were used, the latter allowing the mechanical and electromechanical properties of lysozyme crystals to be mapped simultaneously. Our earlier findings [14] have suggested that monoclinic crystals of lysozyme may be polarizable. Here, we investigate this further using SS-PFM to probe for ferroelectricity in tetragonal crystals of lysozyme.

\section{Methods}

\subsection{Preparation of tetragonal aggregate films of lysozyme}

Crystalline aggregate films of lysozyme were prepared by modifying a Hampton Research crystallization protocol [19]. Briefly, aggregate films of tetragonal lysozyme crystals were prepared by reconstituting lysozyme powder (Sigma-Aldrich, Catalogue Number 62971-50G-F; used without further purification) in sodium acetate $(50 \mathrm{mM}$, $\mathrm{pH} 4.6)$ to a concentration of $100 \mathrm{mg} / \mathrm{mL}$. Glycerol was added to prevent the films from cracking during drying. The glycerol was first diluted to $50 \%$ in DI water for ease of pipetting. Typically, $1-2 \mu \mathrm{L}$ of $50 \%$ glycerol was added per $100 \mu \mathrm{L}$ of protein solution. Then, $100 \mu \mathrm{L}$ of the final protein solution was deposited onto the conductive side of an ITO-coated glass slide and left to dry overnight at $20^{\circ} \mathrm{C}$ in a temperature-controlled room. Crystallization occurred as the films dried. The films represented noncrystalline lysozyme with inclusions of tetragonal crystals of lysozyme.

\subsection{Piezoresponse force microscopy}

In order to characterize the piezoelectric properties of the lysozyme crystals, both contact and Hybrid PFM modes (Ntegra Aura, NT-MDT) were used. Typically, during contact PFM mode, an alternating voltage, $\mathrm{V}_{\mathrm{AC}}$, with a frequency of $20 \mathrm{kHz}$ and amplitude of $1 \mathrm{~V}, 5 \mathrm{~V}$, or $10 \mathrm{~V}$ was applied to the sample via a conductive platinum-coated tip (CSG30/Pt, NT-MDT, force constant $0.6 \mathrm{~N} / \mathrm{m}$, resonant frequency $48 \mathrm{kHz}$ ). The PFM system is fitted with an 
optical microscope, allowing the position of the tip to be adjusted so that it landed either directly on the surface of individual lysozyme crystal or on the area between crystals as desired.

Hybrid mode measurements were implemented via an external HD controller (NTMDT). For Hybrid-AFM measurements, the frequency and amplitude of tip oscillations were set to $1 \mathrm{kHz}$ and $50 \mathrm{~nm}$, respectively. For Hybrid-PFM measurements, the frequency of $\mathrm{V}_{\mathrm{AC}}$ was $10 \mathrm{kHz}$ and the amplitude ranged between $1 \mathrm{~V}$ and $10 \mathrm{~V}$.

Functional analysis, i.e. piezoresponse activity mapping, was carried out using external software (LabVIEW and Python scripts) and external equipment. The equipment included a function generator (Yokogawa FG110), a wideband amplifier (Krohn-hite 7602M), a lock-in amplifier (Stanford Research SR830), and a signal access module (NT-MDT). The piezoresponse and magnitude of hysteresis loop were calculated at each pixel $(1 \mu \mathrm{m}$ movement of the cantilever).

Quantitative PFM measurements were realized by performing PFM at several points across the surface of the lysozyme crystal. The magnitude of the piezoresponse (in units of Ampere) was converted to units of meters using the inverse-optical-sensitivity (IOS) coefficient of the system. The IOS coefficient is calculated from the slope of a force-distance curve performed on a hard substrate. In this case, the IOS coefficient was $0.03 \mathrm{nA} \mathrm{nm}^{-1}$. The magnitude of the piezoresponse per voltage applied gave a quantitative measure of the converse piezoelectric effect in tetragonal crystals of lysozyme.

\section{Results and discussion}

PFM employs the converse piezoelectric effect by applying an electric field via a conductive atomic force microscope (AFM) tip that scans the sample while monitoring the mechanical deformation of the material. This method simultaneously measures the vertical piezoresponse signal (VPFM, Fig. 1g) and the lateral one (LPFM, Fig. 1h), which correspond to an out-of-plane and in-plane response, respectively.

First, contact mode PFM measurements were done in a stepwise manner, where a series of three scans were applied to an area of the tetragonal single crystal of lysozyme to determine if the crystal was polarizable. The first scan $\left(10 \times 10 \mu \mathrm{m}^{2}\right)$ applied $10 \mathrm{~V}$ of bias, the second $\left(40 \times 40 \mu \mathrm{m}^{2}\right)$ applied $5 \mathrm{~V}$ around the first scan area, and the final scan $(60 \times$ $60 \mu \mathrm{m}^{2}$ ) applied a few millivolts of bias around the previous two scan areas. The surface of the sample was affected during the first and second scans, as seen in the topography images (Fig. 1a,b). The lower voltage used in the third scan was less destructive and did not appear to influence substantially the topographical features. The polarizing effect of the bias field applied in the first two scans is evident in the third scan (Fig. 1c-f), indicating that the protein can be polarized with the application of an external electric field.

The tetragonal lysozyme crystal showed an in-plane, LPFM piezoresponse (Fig. 1e,f), which is expected as point group 422 allows shear piezoelectricity. Surprisingly, the out-ofplane component of piezoresponse (VPFM) has been detected simultaneously with the LPFM signal (Fig. 1c,d). LPFM is sensitive to the surface displacements perpendicular to the tip's cantilever axis; therefore, it is expected to demonstrate orientation dependence. The VPFM response combines contributions from longitudinal, transverse, and shear components of piezoelectricity. A finite non-zero VPFM response can be obtained from shear piezoelectricity due to the off-vertical approach of the PFM probe [10]. A buckling vibration, 

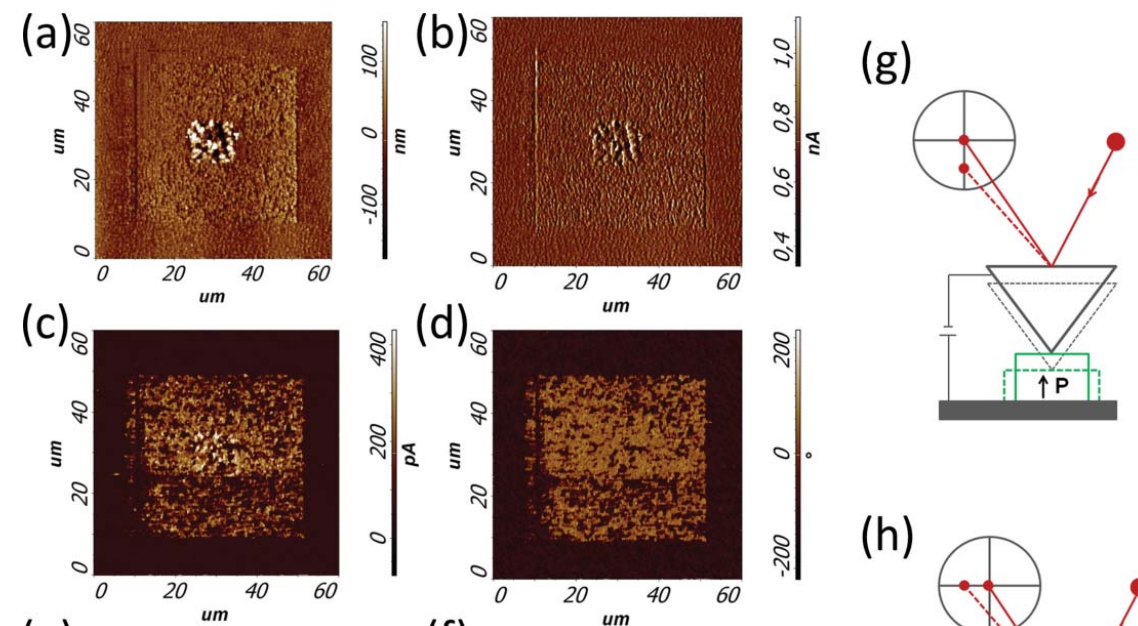

(h)

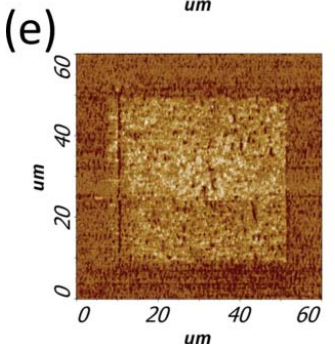

(f)
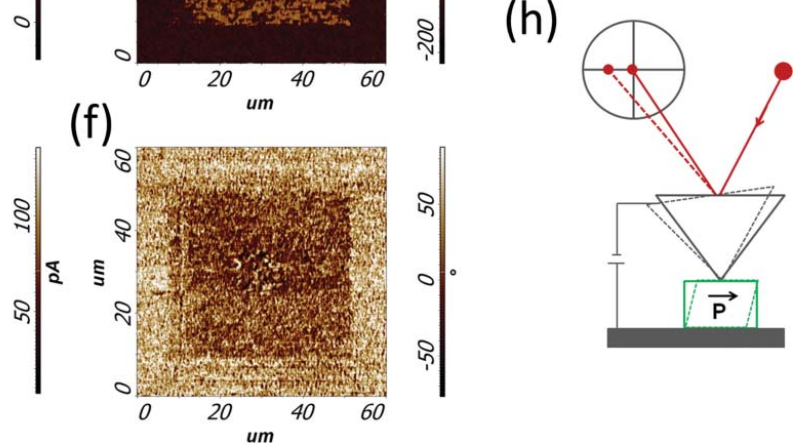

Figure 1. PFM response after biasing a tetragonal lysozyme crystal showing (a) height, (b) deflection, (c) out-of-plane piezoresponse magnitude and (d) phase, (e) in-plane piezoresponse magnitude and (f) phase images. Schematics of vertical $(\mathrm{g})$ and lateral $(\mathrm{h})$ PFM techniques.

caused by in-plane surface displacements and transmitted through frictional forces, can also contribute to the VPFM signal. For example, Harnagea and co-workers [10] observed a direct dependence of both VPFM and LPFM on the angle between the cantilever and fibril axis of collagen fibers.

The VPFM response of tetragonal lysozyme crystals observed here might not have originated from cantilever buckling or from an orientation dependence with respect to the sample due to the off-vertical approach of the PFM probe [20]. An alternative argument to explain the observation of longitudinal piezoelectricity here may be the fact that the symmetry of the crystal is lower than point group 422 (D4); i.e., it may be better described by the point group 4 (C4 symmetry). This type of symmetry lowering has been encountered in bone, which was originally thought to belong to point group 622 (D6 symmetry) [21]. Observations of non-shear piezoelectricity [22] and pyroelectricity [23] in bone prompted its reassignment to the point group 6 (C6 symmetry), which allows both longitudinal and shear piezoelectricity [7]. Similarly, symmetry lowering to monoclinic point group 2 (C2 symmetry) has been reported for wood [24], which had originally been thought of as belonging to point group 622 (D6 symmetry) [25].

Symmetry lowering in crystals of lysozyme has been reported previously. Yamada et al. have shown that above $950 \mathrm{MPa}$ crystals of lysozyme undergo a phase transition from point group 422 to the lower symmetry of point group 4 [26]. While elevated pressure could not have caused symmetry lowering in this study as it was conducting at atmospheric pressure, 
(a)

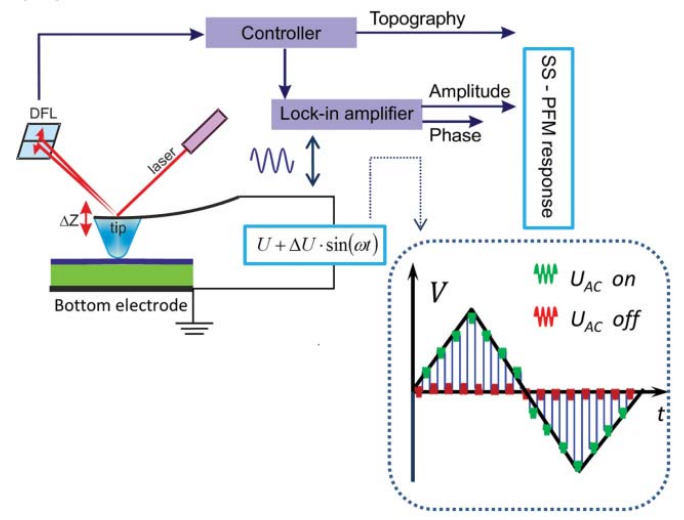

(b)

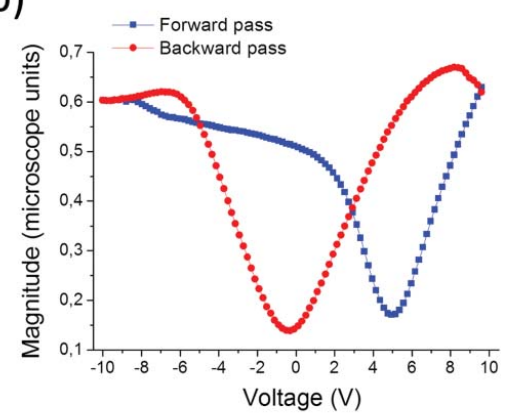

(c)

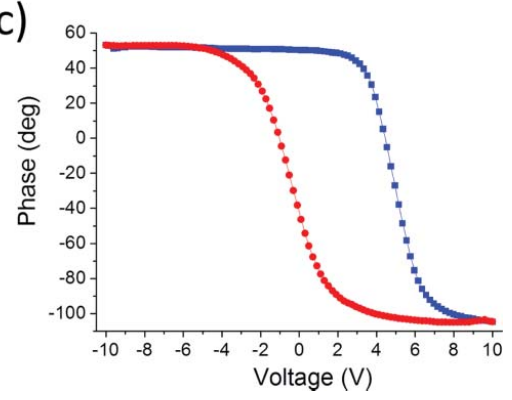

Figure 2. (a) Schematic of SS-PFM measurement technique used to demonstrate characteristic (b) ferroelectric magnitude and (c) phase behaviour in a tetragonal single crystal of lysozyme.

the substrate may strongly influence the lysozyme crystals during growth [27, 28]. We suggest that the substrate may restrict $3 \mathrm{D}$ growth and alter the overall symmetry of the crystals.

The possible lowering of symmetry in lysozyme films to point group 4 (C4 symmetry) is important as it implies the presence of a permanent polarization in the structure. A material with this symmetry can demonstrate pyroelectricity and potentially ferroelectricity if that polarization was switchable. The results already presented in Figure 1 suggest a possible repolarization of electric states within lysozyme in response to an external electric field, indicating that the protein is piezoelectric and ferroelectric.

To reaffirm the ferroelectric nature, we performed switching-spectroscopy PFM measurements (SS-PFM, Fig. 2a), which applies voltage pulses to a specific area of the sample via the conductive AFM probe. If a sample is ferroelectric, the voltage pulses cause the spontaneous polarization of the sample underneath the tip to switch. The characteristic hysteresis loops (Fig. 2) generated by a step bias are typical of ferroelectric materials but with an asymmetry indicative of the presence of some internal bias within the crystal. We postulate that this internal bias may arise from the bound water forming a so-called hydration layer, which may be difficult to switch from one direction to another due to its strongly bound nature. The amplitude and phase response distinguish the sample as ferroelectric by their butterflyshaped amplitude (Fig. 2b) and switching phase loop (Fig. 2c), respectively.

Admittedly, the above results are not free from artifacts associated with carrying out conventional contact mode PFM experiments on soft, hydrated samples such as lysozyme films. To overcome the difficulty of sample damage during PFM, we have also used Hybrid Mode (HD) (HD-AFM ${ }^{\mathrm{TM}}$ Mode, NT-MDT), which is a multifunctional mode that allows simultaneous measurements of multiple physical properties (up to a number of 10) from a single 
AFM scan. HD mode allows measuring physical signals (piezoresponse, topography) and electrical signals (spreading resistance, current), as well as mapping of mechanical properties (Young's modulus, stiffness, adhesion force). The experiment was started on Area type 1, which is non-crystalline and flat and considered to be easier for PFM measurements (Fig. 3). Figure 4a-d shows the simultaneous measurements of topography, stiffness, adhesion, and out-of-plane piezoresponse, respectively, using Hybrid mode AFM on Area type 1. HD-PFM was performed with $5 \mathrm{~V}$ of bias voltage and the piezoresponse was displayed as the product of the magnitude and phase signals. The piezoresponse shows a clear correlation with the adhesion force response, while both of these are distinct from the topography. The topography, however, has influenced the mechanical stiffness (Young's module) response as shown in Figure 4a,b.

Functional analysis, i.e. piezoresponse activity mapping, was performed in contact PFM mode using additional external hardware and software (see Methods) to map the piezoelectric hysteresis loop behavior at each pixel. The size of each pixel corresponds to a $1 \mu \mathrm{m}$ movement of the cantilever. The piezoresponse activity mapping (Fig. 4e) shows that the areas with highest responses quite accurately match the areas with strong piezoresponse and adhesion response. This indicates that the non-crystalline form of lysozyme is polar in nature and is responsible for lysozyme's adhesion response. Higher resolution PFM scans and SS-PFM (Fig. 5) were performed on the areas that showed high mapping response activity. The area selected is indicated by the white box in Figure 4a. With the resolution increased, the topography and stiffness responses are observed to slightly influence the piezoresponse. The area circled in Figure 5a showed the highest piezoresponse. Moreover, several phases have appeared within the domains, which manifest themselves in the stiffness and adhesion response scans (Fig. 5b,c).

To investigate these features further, piezoelectric loops were obtained at two different points, labeled Point 1 and Point 2 in Figure 5c. From the loop analyses (Fig. 5 Figure e) the

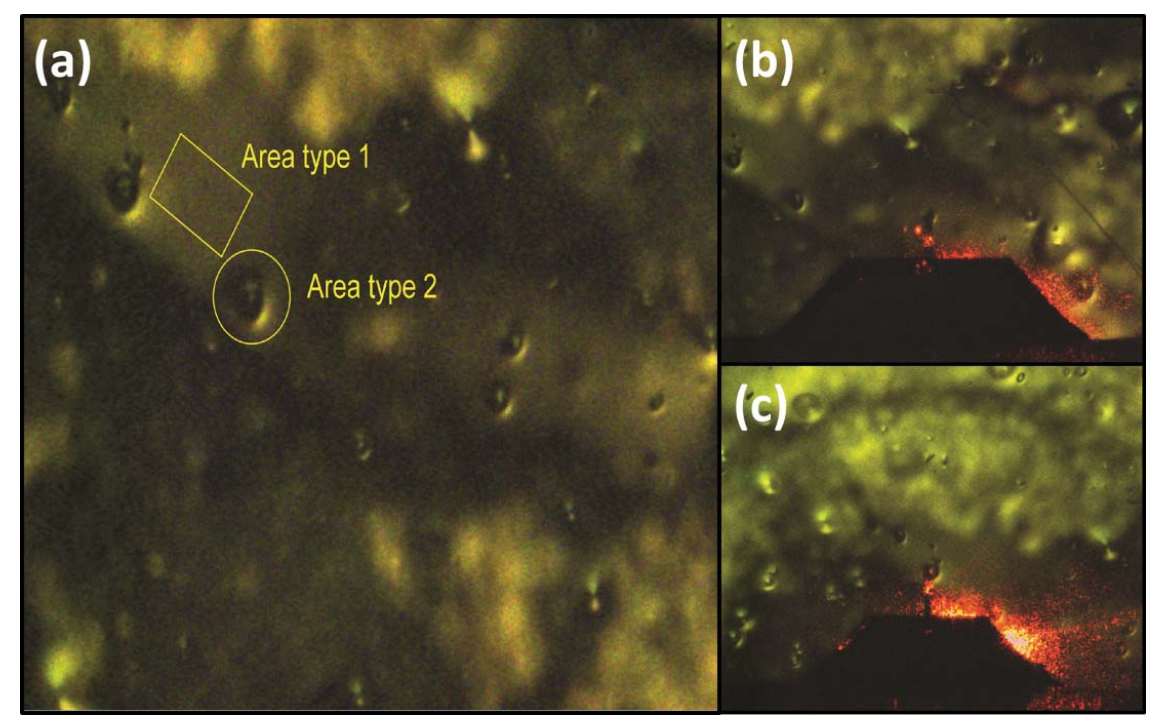

Figure 3. (a) Optical images of the surface of a lysozyme crystalline aggregate film indicating the two areas selected for Hybrid PFM scanning. The tip was moved to Area type 1 (b) to investigate non-crystalline lysozyme and then to Area type 2 (c) to investigate a tetragonal crystal of lysozyme. 
(a)

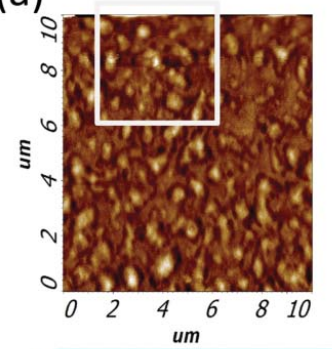

(b)

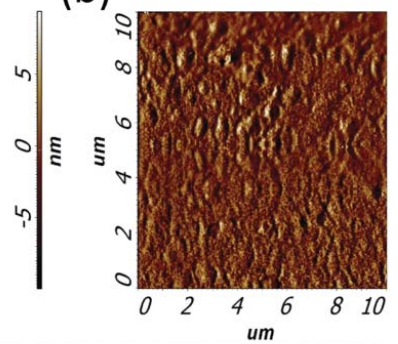

(c)

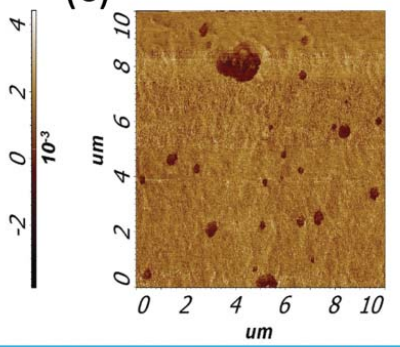

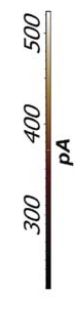
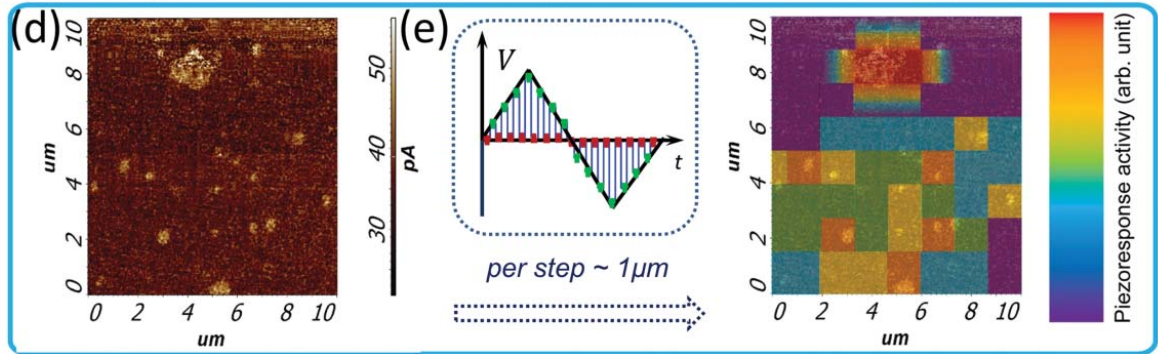

Figure 4. Hybrid mode electrical and mechanical properties images of lysozyme obtained at Area type 1: (a) Topography, boxed area indicates area selected for higher resolution analysis, (b) stiffness response (Young's modulus), (c) adhesion force, (d) out-of-plane PFM response [magnitude $\times$ phase], (e) mapping of the piezoelectric hysteresis loop at each tip step $(1 \mu \mathrm{m})$.

differences in these phases are evident. For example, a magnitude hysteresis and an open phase loops are shown at Point 1 . On the other hand, at Point 2, the magnitude hysteresis loop is narrower and the phase loop is of a closed nature. These kinds of hysteresis behavior could be related to a ferroelectric behavior of the first point and a non-ferroelectric piezoelectric behavior of the second point. Thus, we considered that two different phases may coexist in lysozyme within a given ferroelectric domain.

(a)

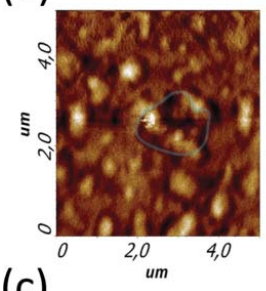

(c)

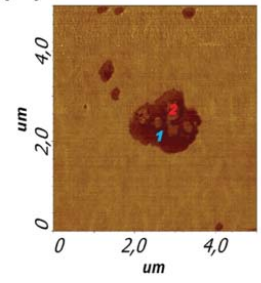

(b)

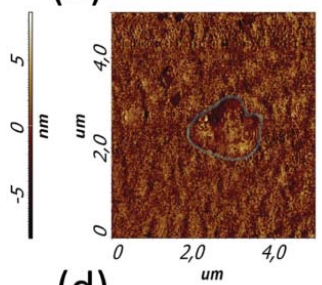

(d)

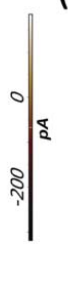

(e)

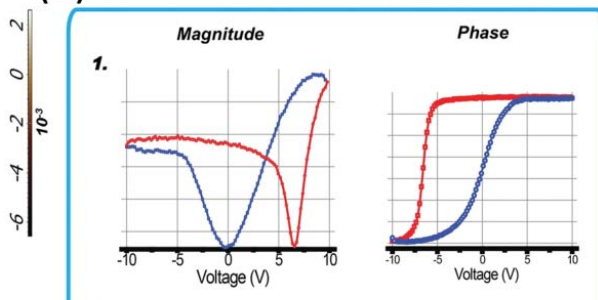

2.

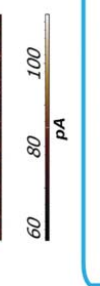

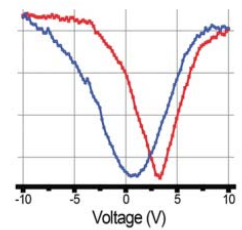

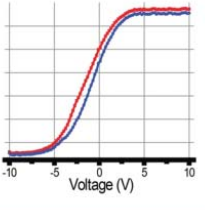

Figure 5. Higher resolution hybrid mode images of the Area type 1: (a) Topography, (b) stiffness (Young's module), (c) adhesion force, (d) out-of-plane PFM response [magnitude $\times$ phase], (e) the ferroelectric magnitude and phase loops corresponding to points 1 and 2 indicated in (c). 

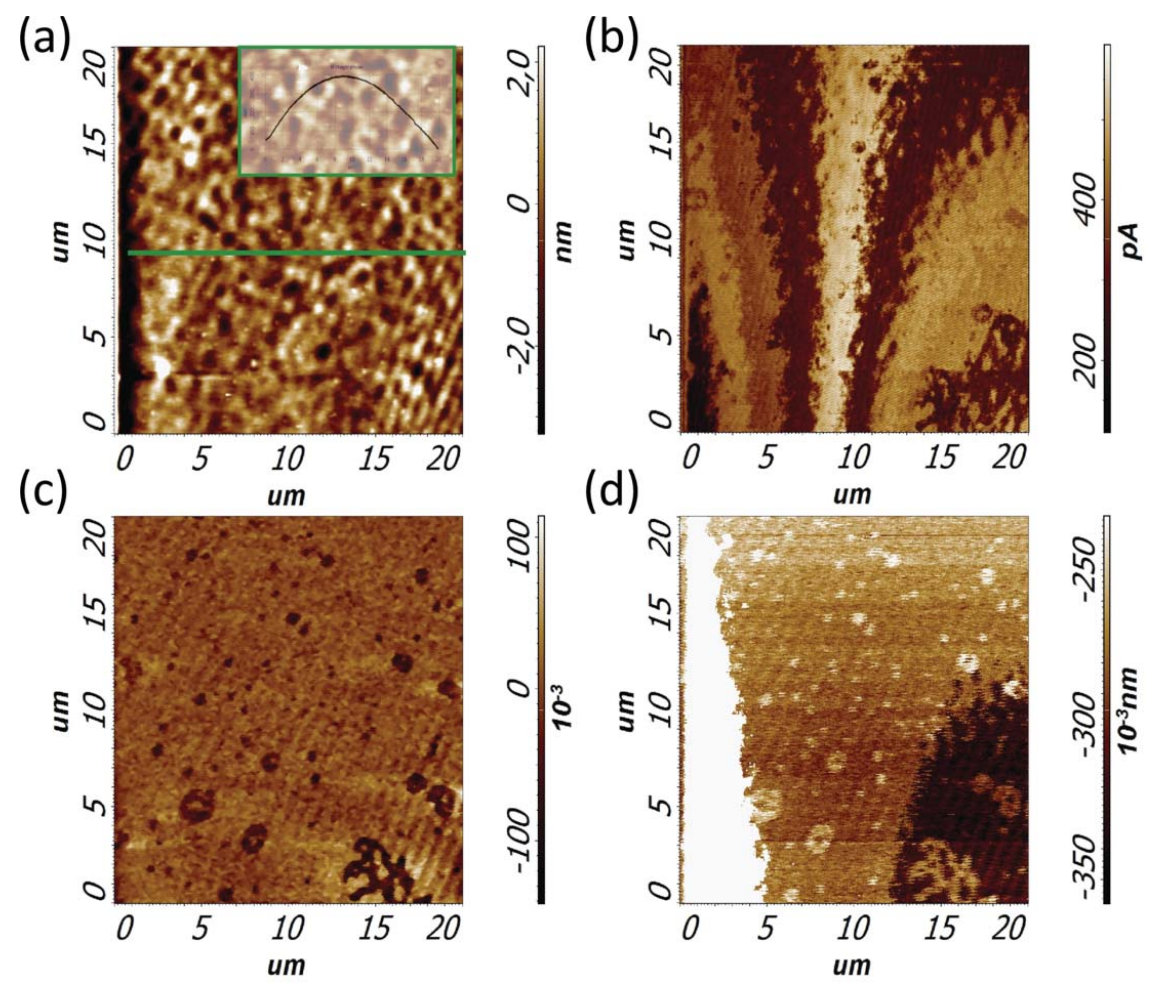

Figure 6. Hybrid mode electrical and mechanical properties scan images of lysozyme obtained at Area type 2: (a) Topography; inset shows the height profile of the green cross-sectional line, (b) out-of-plane PFM response [magnitude $\times$ phase], (c) adhesion force, (d) stiffness response (Young's modulus).

Hybrid mode PFM was employed also in an area dominated by the presence of a single tetragonal microcrystal (Fig. 6). Figure 6a shows that the topography in this area has a highly curved profile (around $500 \mathrm{~nm}$ ). The banded contrast in the piezoresponse image (Fig. 6b) may be due to tip buckling across the curved area scanned. This supposition was also confirmed by the stiffness response data (Fig. 6d). The adhesion response shows that the viscoelastic properties are homogenous on a granular surface (Fig. 6c). Furthermore, the adhesion response manifests features (i.e., small circular areas of dark contrast), which we argue are similar to those observed in the PFM response in Area type 1 (Fig. 3a,c) with dualphase domain structures. This confirms piezoresponse in both non-crystalline and single tetragonal crystal forms of lysozyme.

Lastly, quantitative measurements of tetragonal lysozyme were performed by sweeping a voltage from $0 \mathrm{~V}$ to $10 \mathrm{~V}$ at several points on the surface of the crystal and monitoring the magnitude of the piezoresponse. Figure 7 shows the average magnitude of the piezoresponse of tetragonal crystal of lysozyme obtained over six PFM points. The plot does not go through the origin, indicating that there is some contribution from electrostatic effects. Electrostatic interactions are also likely to be the cause of the non-linear piezoresponse. The piezoelectric coefficient is determined from the linear part of the plot indicated and the IOS coefficient. The piezoelectric coefficient of tetragonal lysozyme as measured by $\mathrm{PFM}$ is $19.3 \mathrm{pm} \mathrm{V}^{-1}$. 


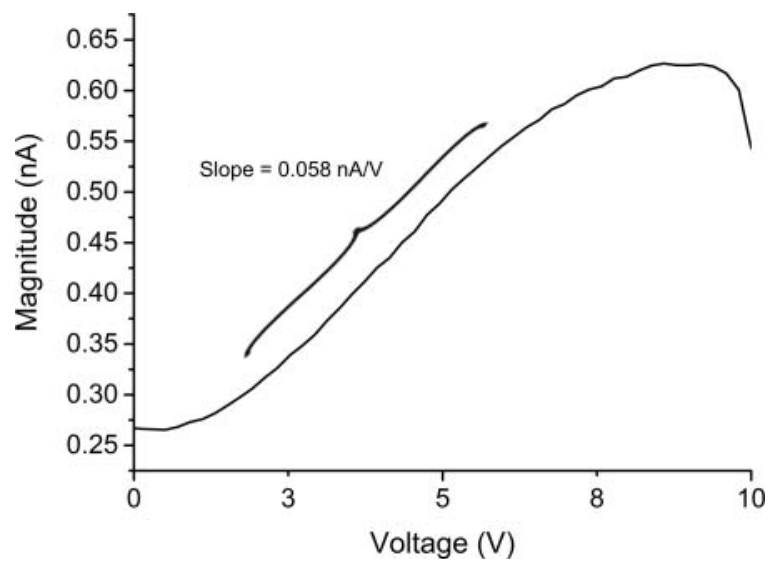

Figure 7. SS-PFM performed on tetragonal lysozyme showing the average magnitude of the piezoresponse to an applied voltage. The piezoelectric coefficient is determined from the slope of the linear part of the plot.

\section{Conclusion}

In summary, we have observed piezoelectricity in aggregate films of lysozyme in areas of noncrystalline lysozyme, as well as in individual tetragonal crystals of lysozyme. The converse piezoelectric effect was quantified using PFM as approximately $19.3 \mathrm{pm} \mathrm{V}^{-1}$. The observation of longitudinal piezoelectricity and ferroelectricity indicated that the protein might be of lower symmetry (point group 4) than that typically assigned for tetragonal lysozyme (point group 422) from X-ray crystallography. Such a lowering of symmetry allowed polarization switching using a local probe and the behavior is similar to asymmetric ferroelectricity observed in inorganic crystals [29]. Using Hybrid PFM, the piezoresponse and mechanical properties of lysozyme were mapped simultaneously. The fact that non-crystalline form of lysozyme shows a switchable polarization concurrent with its adhesion response may indicate that lysozyme uses its electrical polarization to influence the binding of water to create its hydration layer. The asymmetric, local ferroelectric hysteresis loop we have observed may indicate the presence of a mechanism that supports the transduction of electrochemical signals involved in lysozymes solubility and secretion in cells. Compared to fibrous proteins, globular proteins are soluble and crystallize readily, which may have important implications for realizable technical applications.

\section{Funding}

Funding from the Irish Research Council EMBARK Postgraduate Scholarship (RS/2012/337) to A.S. is acknowledged. M.S.I. is grateful to FCT for financial support through the project MATIS - Materiais e Tecnologias Industriais Sustentáveis (CENTRO-01-0145-FEDER-000014). Part of this study was also facilitated by a HEA grant under the Programme for Research in Third-Level Institutions (PRTLI 5) to the University of Limerick. A.L.K. acknowledges the CICECO-Aveiro Institute of Materials (Ref. FCT UID/CTM/50011/2013) financed by national funds through the FCT/MEC and, when applicable, cofinanced by FEDER under the PT2020 Partnership Agreement.

\section{Author contribution}

A. S., M. R. N., and T. S. planned and prepared the non-crystalline and crystalline aggregate films of lysozyme. M. I. and A. S. performed all SS-PFM measurements and piezoresponse activity mapping. 
A. K, S. A. M. T., T. S, C. S., and A. A. G. were directly involved in the strategic planning, data analysis, and discussion. A. S., M. I., and S. A. M. T. wrote the manuscript and all authors were involved in the scientific discussion and proofreading prior to submission.

\section{References}

[1] E. Seyedhosseini, A. L. Kholkin, D. Vasileva, A. Nuraeva, S. Vasilev, P. S. Zelenovskiy, V. Ya. Shur: Patterning and nanoscale characterization of ferroelectric amino acid beta-glycine. In: Joint IEEE International Symposium on the Applications of Ferroelectric, International Symposium on Integrated Functionalities and Piezoelectric Force Microscopy Workshop (ISAF/ISIF/PFM). IEEE; 2015 May: 207-210.

[2] E. Seyedhosseini, I. Bdikin, M. Ivanov, D. Vasileva, A. Kudryavtsev, B. Rodriguez, et al: Tipinduced domain structures and polarization switching in ferroelectric amino acid glycine. J Appl Phys. 2015; 118(7): 072008.

[3] A. L. Kholkin, N. Amdursky, I. Bdikin, E. Gazit, G. Rosenman: Strong piezoelectricity in bioinspired peptide nanotubes. ACS Nano. 2010; 4: 610-614.

[4] V. S. Bystrov, I. Bdikin, A. Heredia, R. C. Pullar, E. Mishina, A. S. Sigov, et al: Piezoelectricity and ferroelectricity in biomaterials: from proteins to self-assembled peptide nanotubes. In: G. Ciofani, A. Menciassi, eds. Piezoelectric nanomaterials for biomedical applications: Springer; 2012: $187-211$.

[5] B. Y. Lee, J. Zhang, C. Zueger, W.-J. Chung, S. Y. Yoo, E. Wang, et al: Virus-based piezoelectric energy generation. Nat Nanotechnol. 2012; 7: 351-356.

[6] J. Curie, P. Curie: Développement par compression de l'électricité polaire dans les cristaux hémièdres à faces inclinées. B Soc Minéral Fr. 1880; 3: 90-93.

[7] Sirotin IUrI, Shaskol'skaia MP: Fundamentals of crystal physics. Mir Publishers; 1982.

[8] D. Denning, J. I. Kilpatrick, E. Fukada, N. Zhang, S. Habelitz, A. Fertala, et al: The piezoelectric tensor of collagen fibrils determined at the nanoscale. ACS Biomater Sci Eng. 2017; 3: 929-935.

[9] M. Minary-Jolandan, M.-F. Yu: Uncovering nanoscale electromechanical heterogeneity in the subfibrillar structure of collagen fibrils responsible for the piezoelectricity of bone. ACS Nano. 2009; 3: 1859-1863.

[10] C. Harnagea, M. Vallières, C. P. Pfeffer, D. Wu, B. R. Olsen, A. Pignolet, et al: Two-dimensional nanoscale structural and functional imaging in individual collagen type I fibrils. Biophys J. 2010; 98: 3070-3077.

[11] M. Minary-Jolandan, M.-F. Yu: Nanoscale characterization of isolated individual type I collagen fibrils: polarization and piezoelectricity. Nanotechnol. 2009; 20: 085706.

[12] Y. Liu, H.-L. Cai, M. Zelisko, Y. Wang, J. Sun, F. Yan, et al: Ferroelectric switching of elastin. Proc Natl Acad Sci. 2014; 111: E2780-E2786.

[13] Y. Liu, Y. Wang, M.-J. Chow, N. Q. Chen, F. Ma, Y. Zhang, et al: Glucose suppresses biological ferroelectricity in aortic elastin. Phys Rev Lett. 2013; 110: 168101.

[14] A. Stapleton, M. R. Noor, T. Soulimane, S. A. Tofail: Physiological role of piezoelectricity in biological building blocks. In: S. A. M. Tofail, J. Bauer, eds. Electrically active materials for medical devices: World Scientific; 2016: 237-251.

[15] C. Brinkmann, M. S. Weiss, E. Weckert: The structure of the hexagonal crystal form of hen eggwhite lysozyme. Acta Crystallogr D. 2006; 62: 349-355.

[16] I. Danielewicz-Ferchmin, E. M. Banachowicz, A. R. Ferchmin: Role of electromechanical and mechanoelectric effects in protein hydration under hydrostatic pressure. Phys Chem Chem Phys. 2011; 13: 17722-17728.

[17] M. G. Ortore, F. Spinozzi, P. Mariani, A. Paciaroni, L. R. Barbosa, H. Amenitsch, et al: Combining structure and dynamics: non-denaturing high-pressure effect on lysozyme in solution. J Roy Soc Interface. 2009; 6: S619-S634.

[18] S. V. Kalinin, B. J. Rodriguez, S. Jesse, K. Seal, R. Proksch, S. Hohlbauch, et al: Towards local electromechanical probing of cellular and biomolecular systems in a liquid environment. Nanotechnology. 2007; 18: 424020. 
[19] Hampton Research, Lysozyme Online User Guide, Crystallization Experiments. 2014.

[20] S. V. Kalinin, B. J. Rodriguez, S. Jesse, J. Shin, A. P. Baddorf, P. Gupta, et al: Vector piezoresponse force microscopy. Microscopy and Microanalysis. 2006; 12: 206-220.

[21] E. Fukada, I. Yasuda: On the piezoelectric effect of bone. J Phys Soc Japan. 1957; 12: 1158-1162.

[22] E. Fukada, I. Yasuda: Piezoelectric effects in collagen. Jap J Appl Phys. 1964; 3: 117.

[23] S. B. Lang: Pyroelectric effect in bone and tendon. Nature. 1966; 212: 704-705.

[24] E. Fukada: Piezoelectricity of wood. J Phys Soc Japan. 1955; 10: 149-154.

[25] N. Hirai, N. Sobue, M. Date: New piezoelectric moduli of wood: d 31 and d 32. J Wood Science. 2011; 57: 1-6.

[26] H. Yamada, T. Nagae, N. Watanabe: High-pressure protein crystallography of hen egg-white lysozyme. Acta Crystallographica Section D: Biological Crystallography. 2015; 71: 742-753.

[27] L.-H. Sun, C.-Y. Xu, F. Yu, S.-X. Tao, J. Li, H. Zhou, et al: Epitaxial growth of trichosanthin protein crystals on mica surface. Crystal Growth \& Design. 2010; 10: 2766-2769.

[28] L. Rong, H. Komatsu, M. Natsuisaka, S. Yoda: Epitaxial nucleation of protein crystal on poly-Llysine modified surface. Jap J Appl Phys. 2001; 40: 6677.

[29] K. Abe, N. Yanase, T. Yasumoto, T. Kawakubo: Nonswitching layer model for voltage shift phenomena in heteroepitaxial barium titanate thin films. Jap J App Phys. 2002; 41: 6065. 